\title{
A Clinicopathological Study of Breast Carcinoma- Comparison of Triple Negative with Non-Triple Negative Breast Cancers
}

\author{
Khandekar Prachi, Vimal Shruti*, Gore Charusheela, Jain Kanika, Dharwadker Arpana, Vishwanathan Vidya \\ Department of Pathology, Dr. D. Y. Patil Medical College, Hospital and Research Centre, Dr. D Y Patil Vidyapeeth, Pimpri ,Pune, Maharashtra, India
}

DOI: $\underline{10.36348 / \mathrm{sjpm} .2020 . \mathrm{v} 05 \mathrm{i} 02.003}$

| Received: 20.01.2020 | Accepted: 27.01.2020 | Published: 11.02 .2020

*Corresponding author: Dr. Shruti Vimal

\section{Abstract}

Introduction: The most common malignancy encountered, in current setup is breast cancer in females. It stands second, as the most common cause, of death caused by cancers in developed countries. The diagnosis of the variants of breast cancer can be done with aid of immunohistochemistry (IHC) that is expression of ER, PR, Her2/Neu, and this has led to application of multiple systemic therapeutic strategies. The purpose of this study is to find the proportion of triple negative breast cancers in the study group and to compare the clinical features, natural history and outcome of Triple Negative Breast Cancer (TNBC) to other non-triple variants of breast cancers. Materials and methods: Analysis of retrospective clinical database was performed for 100 cases for the duration of 3 years (2017-2019). Clinical, histopathological and IHC patterns were compared under various headings. Results: Significant difference was found between the two groups in the variables such as age of diagnosis, tumor size, grade, lymphatic spread and prognosis. The patterns of reference amongst them show qualitative difference with triple negative group in view of risk of recurrence which peaked at 4 years and declined rapidly. On the contrary the others presented with constant risk of recurrence over a period of time. Conclusion: Triple Negative Breast Cancer has a more aggressive clinical setup as compared to others, although the findings are transient. The morphological characteristics alone cannot be used to classify breast cancers in two subtypes with different prognosis.

Keywords: Breast Cancer, Immunohistochemistry, Triple Negative, Triple positive, Comedo necrosis.

Copyright @ 2020: This is an open-access article distributed under the terms of the Creative Commons Attribution license which permits unrestricted use, distribution, and reproduction in any medium for non-commercial use (NonCommercial, or CC-BY-NC) provided the original author and sources are credited.

\section{INTRODUCTION}

Breast cancers are the most common neoplasm being encountered in the new era accounting for approximately one fourth of all cancers in female's world- wide and $27 \%$ cancers in developed countries with western lifestyle [1]. Men also present with breast cancer but its more than 100 times more common in women than in men. It usually has a bad prognosis invariably because of the delay in diagnosis [3, 17]. Carcinoma breast occurs in any cells of the mammary glands and exhibit wide scope of morphological, immunohistochemical profile and unique histopathological subtypes with different clinical course and outcome.

\footnotetext{
The study was undertaken to compare the different histo-morphological findings with immunohistochemical parameters and presence of necrosis in the various categories.
}

\section{MATERIALS AND METHODS}

A retrospective study of 102 diagnosed cases of carcinoma breasts on histopathological examination. The patients had reported to our tertiary care center Dept of Pathology D Y Patil Medical College and Research Centre between the duration of 2017 to 2019. All the cases were reported on the basis of histopathological examination and hormone receptors of ER, PR and Her 2 markers. The clinical details of the patient were taken from the medical records department. The histopathology and IHC slides were prepared according to the general guidelines and were examined independently by two pathologists for morphological examination and lympho- vascular invasions were diagnosed on the basis of Modified Bloom Richardson Scoring. The IHC antibodies used were ER (Thermo Fischer Scientific), PR (Thermo Fischer Scientific) and Her 2 (Thermo Fischer Scientific). The ER PR pattern scoring was done on the criterion of Allred Score and the final score was concluded on the basis of proportional score and intensity score. False negative assays were avoided and 
for the same internal scores were used. For Her 2 more than $10 \%$ cells showing complete circumferential membrane staining that is complete and intense were considered positive i.e. $(3+)$.

Tumors showing positivity for ER, PR and Her 2 (triple positive), for any of the two markers (double positive), single marker (single positive) and not showing positivity at all were labelled as triple negative. The groups were compared on the basis of cytomorphological patterns, lymphatic and vascular invasions, necrosis, lymphatic metastasis, Bloom Richardson scoring and grading of the lesion.

\section{RESULTS}

The lesions were broadly classified according to tumor estrogen receptors, progesterone receptors and human epidermal growth factor receptor 2 (Her 2) triple negative, triple positive, double positive and single positive and statistical analysis ( Chi squared test) was done in all with interpretation of the $p$ value. The total number of cases undertaken for the study was 102. Peak incidence of carcinoma breast was noted in the $30^{\text {th }}$ and $40^{\text {th }}$ decades with the mean age of presentation at 48.9 yrs. The median age for presentation triple negative markers was 49.8 yrs. while that of triple, double and single positive marker was 48.5 yrs. On the basis of size of the tumor i.e. $<5 \mathrm{cms}$ or $>5 \mathrm{cms}$ the lesions were classified further on the basis of hormonal response (Table1 and 2).

Table-1: Tumour size compared to the Hormonal receptors

\begin{tabular}{|l|l|}
\hline Classification X & Tumor_size__5cms \\
\hline Classification Y & Hormonal response (IHC markers) \\
\hline
\end{tabular}

\begin{tabular}{|l|l|l|l|}
\hline & Tumor_size & \multicolumn{5}{l}{ 5cms } & \\
\hline Hormonal response & N & Y & \\
\hline Triple Negative & $618.2 \%$ RT & $2781.8 \%$ RT & $33(32.4 \%)$ \\
\hline Triple Positive & $19.1 \%$ RT & $1090.9 \%$ RT & $11(10.8 \%)$ \\
\hline Two positive & $721.2 \%$ RT & $2678.8 \%$ RT & $33(32.4 \%)$ \\
\hline Single positive & $728.0 \%$ RT & $1872.0 \%$ RT & $25(24.5 \%)$ \\
\hline & $21(20.6 \%)$ & $81(79.4 \%)$ & 102 \\
\hline
\end{tabular}

RT: \% of Row Total; CT: \% of Column Total; GT: \% of Grand Total

Show all percentages

Table-2:Chi-squared test with $P$ value for tumor size

\begin{tabular}{|l|l|}
\hline Chi-squared & $\mathbf{1 . 8 5 4}$ \\
\hline DF & 3 \\
\hline Significance level & $\mathrm{P}=0.6032$ \\
\hline
\end{tabular}

Chi-squared test for trend

\begin{tabular}{|l|l|}
\hline Chi-squared (trend) & $\mathbf{0 . 9 5 7}$ \\
\hline DF & 1 \\
\hline Significance level & $\mathrm{P}=0.3279$ \\
\hline
\end{tabular}

On cytomorphological examination a close correlation was noted in the presence of necrosis amongst the four groups. The necrosis noted was mainly geographical and presented with comedo necrosis and central necrosis (Table 3 and 4). Lymphovascular invasions and lymphatic metastasis was assessed in all the groups separately. (Table 5 and 6).

Table-3: Presence of necrosis compared to the Hormonal receptors

\begin{tabular}{|l|l|}
\hline Classification X & Necrosis \\
\hline Classification Y & Hormonal response (IHC markers) \\
\hline
\end{tabular}

\begin{tabular}{|c|c|c|c|}
\hline & \multicolumn{2}{|l|}{ Necrosis } & \\
\hline Hormonal response & $\mathbf{N}$ & $\mathbf{Y}$ & \\
\hline All negatives & $1236.4 \% \mathrm{RT}$ & $2163.6 \% \mathrm{RT}$ & $33(32.4 \%)$ \\
\hline Single positive & $1352.0 \% \mathrm{RT}$ & $1248.0 \% \mathrm{RT}$ & $25(24.5 \%)$ \\
\hline Two positives & $2266.7 \% \mathrm{RT}$ & $1133.3 \% \mathrm{RT}$ & $33(32.4 \%)$ \\
\hline All positives & $872.7 \%$ RT & $327.3 \%$ RT & $11(10.8 \%)$ \\
\hline & $55(53.9 \%)$ & $47(46.1 \%)$ & 102 \\
\hline
\end{tabular}

RT: \% of Row Total; CT: \% of Column Total; GT: \% of Grand Total

$\Gamma$ Show all percentages 
Table-4: Presence of necrosis compared to the Hormonal receptors Chi-squared test

\begin{tabular}{|l|l|}
\hline Chi-squared & $\mathbf{7 . 8 5 5}$ \\
\hline DF & 3 \\
\hline Significance level & $\mathrm{P}=0.0491$ \\
\hline
\end{tabular}

Table-5: Lymphatic Invasion

\begin{tabular}{|l|l|}
\hline Classification X & Lymphatic invasion \\
\hline Classification Y & Hormonal response (IHC markers) \\
\hline
\end{tabular}

\begin{tabular}{|c|c|c|c|}
\hline & \multicolumn{2}{|c|}{ Lymphatic invasion } & \\
\hline Hormonal response & $\mathrm{N}$ & $\mathrm{Y}$ & \\
\hline Triple Negative & $1442.4 \% \mathrm{RT}$ & $1957.6 \% \mathrm{RT}$ & $33(32.4 \%)$ \\
\hline Triple positive & $436.4 \% \mathrm{RT}$ & $763.6 \% \mathrm{RT}$ & $11(10.8 \%)$ \\
\hline Two positives & $1236.4 \% \mathrm{RT}$ & $2163.6 \% \mathrm{RT}$ & $33(32.4 \%)$ \\
\hline Single positive & $1144.0 \% \mathrm{RT}$ & $1456.0 \% \mathrm{RT}$ & $25(24.5 \%)$ \\
\hline & $41(40.2 \%)$ & $61(59.8 \%)$ & 102 \\
\hline
\end{tabular}

RT: \% of Row Total; CT: \% of Column Total; GT: \% of Grand Total

$\ulcorner$ Show all percentages

Table-6: Lymphatic Invasion Chi-squared test

\begin{tabular}{|l|l|}
\hline Chi-squared & $\mathbf{0 . 4 8 7}$ \\
\hline DF & 3 \\
\hline Significance level & $\mathrm{P}=0.9216$ \\
\hline
\end{tabular}

Chi-squared test for trend

\begin{tabular}{|l|l|}
\hline Chi-squared (trend) & $\mathbf{0 . 0 0 0}$ \\
\hline DF & 1 \\
\hline Significance level & $\mathrm{P}=0.9866$ \\
\hline
\end{tabular}

A total of $60.6 \%$ cases in triple negative showed vascular invasion, followed by single positive, double positive and lastly triple positive (table 7 and 8 )

Table-7: Vascular invasion

\begin{tabular}{|l|l|}
\hline Classification X & Vascular invasion \\
\hline Classification Y & Hormonal response (IHC markers) \\
\hline
\end{tabular}

\begin{tabular}{|l|l|l|l|}
\hline & \multicolumn{2}{|l|}{ Vascular invasion } & \\
\hline Hormonal response & $\mathrm{N}$ & $\mathrm{Y}$ & \\
\hline Triple Negatives & $1339.4 \% \mathrm{RT}$ & $2060.6 \% \mathrm{RT}$ & $33(32.4 \%)$ \\
\hline Triple positives & $763.6 \% \mathrm{RT}$ & $436.4 \% \mathrm{RT}$ & $11(10.8 \%)$ \\
\hline Two positives & $1545.5 \% \mathrm{RT}$ & $1854.5 \% \mathrm{RT}$ & $33(32.4 \%)$ \\
\hline Single positive & $832.0 \% \mathrm{RT}$ & $1768.0 \% \mathrm{RT}$ & $25(24.5 \%)$ \\
\hline & $43(42.2 \%)$ & $59(57.8 \%)$ & 102 \\
\hline
\end{tabular}

RT: \% of Row Total; CT: \% of Column Total; GT: \% of Grand Total

$$
\text { Г Show all percentages }
$$

Table-8: Vascular invasion Chi-squared test

\begin{tabular}{|l|l|}
\hline Chi-squared & $\mathbf{3 . 3 8 9}$ \\
\hline DF & 3 \\
\hline Significance level & $\mathrm{P}=0.3354$ \\
\hline
\end{tabular}

Chi-squared test for trend
\begin{tabular}{|l|l|}
\hline Chi-squared (trend) & $\mathbf{0 . 2 7 5}$ \\
\hline DF & 1 \\
\hline Significance level & $\mathrm{P}=0.6002$ \\
\hline
\end{tabular}

Metastatic deposits in the ipsilateral lymph nodes were noted in total of $32.4 \%$ cases of triple negative, $10.8 \%$ of triple positive, $32.4 \%$ of double positive and $24.5 \%$ of single positive. (Table 9 and 10) 
Table-9: Lymphatic Metastasis

\begin{tabular}{|l|l|}
\hline Classification X & Lymph node metastasis \\
\hline Classification Y & Hormonal response (IHC markers) \\
\hline
\end{tabular}

\begin{tabular}{|l|l|l|l|}
\hline & \multicolumn{2}{|l|}{ Lymph node mets } & \\
\hline Hormonal response & $\mathrm{N}$ & $\mathrm{Y}$ & \\
\hline Triple Negatives & $1648.5 \% \mathrm{RT}$ & $1751.5 \% \mathrm{RT}$ & $33(32.4 \%)$ \\
\hline Triple positives & $436.4 \% \mathrm{RT}$ & $763.6 \% \mathrm{RT}$ & $11(10.8 \%)$ \\
\hline Two positives & $1236.4 \% \mathrm{RT}$ & $2163.6 \% \mathrm{RT}$ & $33(32.4 \%)$ \\
\hline Single positives & $1352.0 \% \mathrm{RT}$ & $1248.0 \% \mathrm{RT}$ & $25(24.5 \%)$ \\
\hline & $45(44.1 \%)$ & $57(55.9 \%)$ & 102 \\
\hline
\end{tabular}

RT: \% of Row Total; CT: \% of Column Total; GT: \% of Grand Total $\ulcorner\quad$ Show all percentages

Table-10: Lymphatic Metastasis Chi-squared test

\begin{tabular}{|l|l|}
\hline Chi-squared & $\mathbf{1 . 9 5 8}$ \\
\hline DF & 3 \\
\hline Significance level & $\mathrm{P}=0.5811$ \\
\hline
\end{tabular}

Chi-squared test for trend

\begin{tabular}{|l|l|}
\hline Chi-squared (trend) & $\mathbf{0 . 0 0 0}$ \\
\hline DF & 1 \\
\hline Significance level & $\mathrm{P}=0.9921$ \\
\hline
\end{tabular}

The lesions were graded and assessed according to Modified Bloom Richardson Scoring in both the groups separately. Amongst the triple negative group most of the cases were in Grade II (53.1\%), Grade III (25\%) and Grade I (21.8\%). On morphological examination, majority of the cases were diagnosed as Infiltrating Ductal carcinoma (NOS) were $90.6 \%$, followed by lobular, mixed lobular and ductal, medullary and metaplastic carcinomas $(3.1 \%)$. In triple positive, double positive and single positive group, the lesions were graded as per Modified Bloom Richardson Scoring Grade I (48.5\%), followed by grade II (47.1\%) and lastly grade III $(28.5 \%)$. Majority of the lesions on morphological examination were those of infiltrating ductal carcinoma NOS type (97.1\%). Finally a summary of all the parameters was made to compare and analyse. (Table 11).

Table-11: Summary of statistical tables

\begin{tabular}{|l|l|}
\hline Data & Age \\
\hline Factor codes & Code \\
\hline
\end{tabular}

\begin{tabular}{|l|l|}
\hline Sample size & 102 \\
\hline
\end{tabular}

Levene's test for equality of error variances

\begin{tabular}{|l|l|}
\hline Levene statistic & $\mathbf{0 . 0 9 8 9}$ \\
\hline DF 1 & 3 \\
\hline DF 2 & 98 \\
\hline Significance level & $\mathrm{P}=0.960$ \\
\hline
\end{tabular}

ANOVA

\begin{tabular}{|l|l|l|l|}
\hline Source of variation & Sum of Squares & DF & Mean Square \\
\hline $\begin{array}{l}\text { Between groups } \\
\text { (influence factor) }\end{array}$ & 932.0723 & 3 & 310.6908 \\
\hline $\begin{array}{l}\text { Within groups } \\
\text { (other fluctuations) }\end{array}$ & 14575.8885 & 98 & 148.7336 \\
\hline Total & 15507.9608 & 101 & \\
\hline
\end{tabular}

\begin{tabular}{|l|l|}
\hline F-ratio & $\mathbf{2 . 0 8 9}$ \\
\hline Significance level & $\mathrm{P}=0.107$ \\
\hline
\end{tabular}

\begin{tabular}{|l|l|l|l|}
\hline Factor & n & Mean & SD \\
\hline$(1) 0$ & 33 & 50.0303 & 12.6676 \\
\hline$(2) 1$ & 11 & 56.0000 & 12.3774 \\
\hline$(3) 2$ & 33 & 48.3939 & 11.4617 \\
\hline (4) 3 & 25 & 45.2800 & 12.4248 \\
\hline
\end{tabular}




\begin{tabular}{|l|l|}
\multicolumn{2}{c}{ Residuals } \\
\hline $\begin{array}{l}\text { Shapiro-Wilk test } \\
\text { for Normal distribution }\end{array}$ & $\begin{array}{l}\mathrm{W}=0.9805 \\
\text { accept Normality }(\mathrm{P}=0.1380)\end{array}$ \\
\hline
\end{tabular}

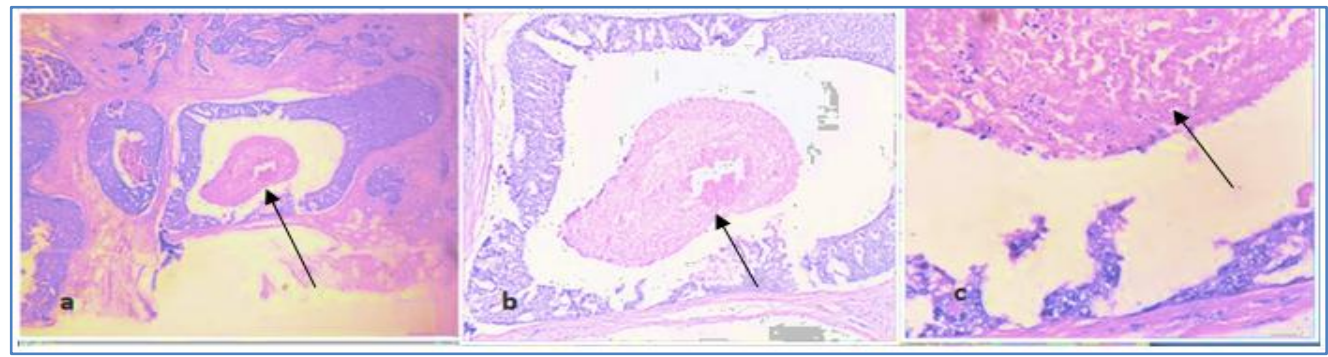

Fig-1: HPE showing area of central necrosis within the ductular epithelial cells shown by arrows in a triple negative breast carcinoma. A) H/E stain scanner view. B) H/E stains 10 xs. c) H/E stain 40x

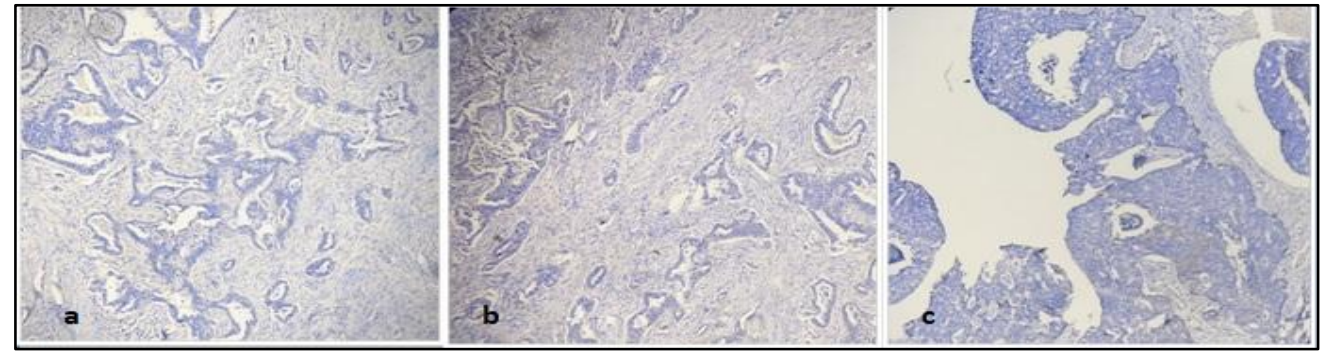

Fig-2: Immunohistochemistry in a triple negative breast carcinoma showing a) ER negative b) PR negative c) HER2/Neu negative

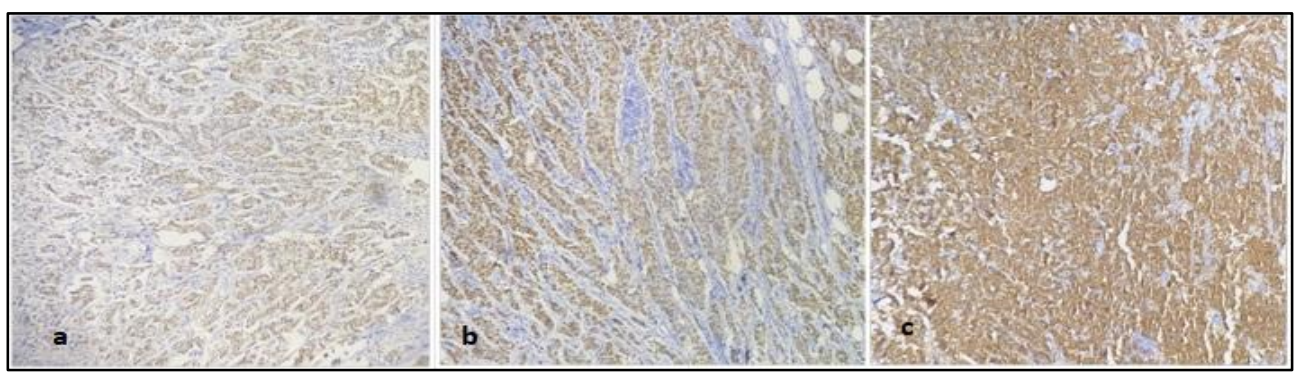

Fig-3: Immunohistochemistry in a triple positive breast carcinoma showing a) ER positive b) PR positive c) HER2/Neu positive

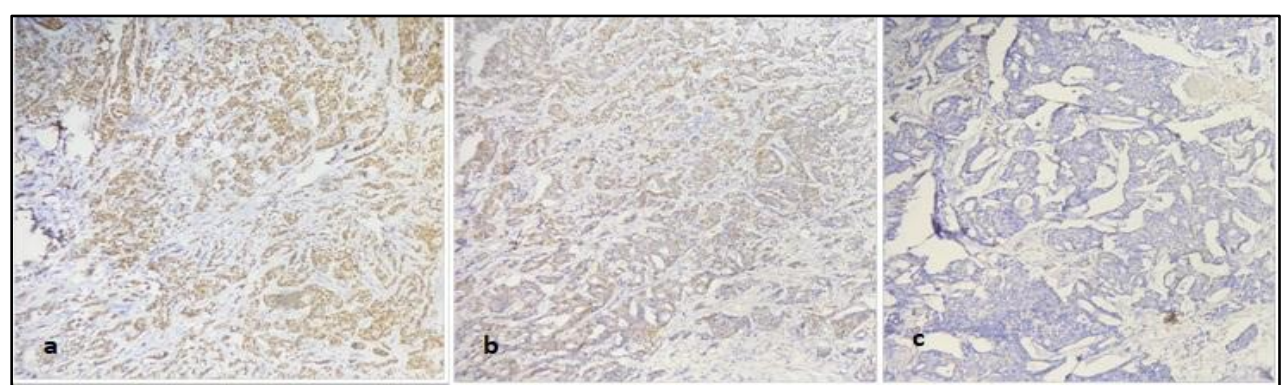

Fig-4: Immunohistochemistry in a breast carcinoma showing a) ER positive b) PR positive c) HER2/Neu negative

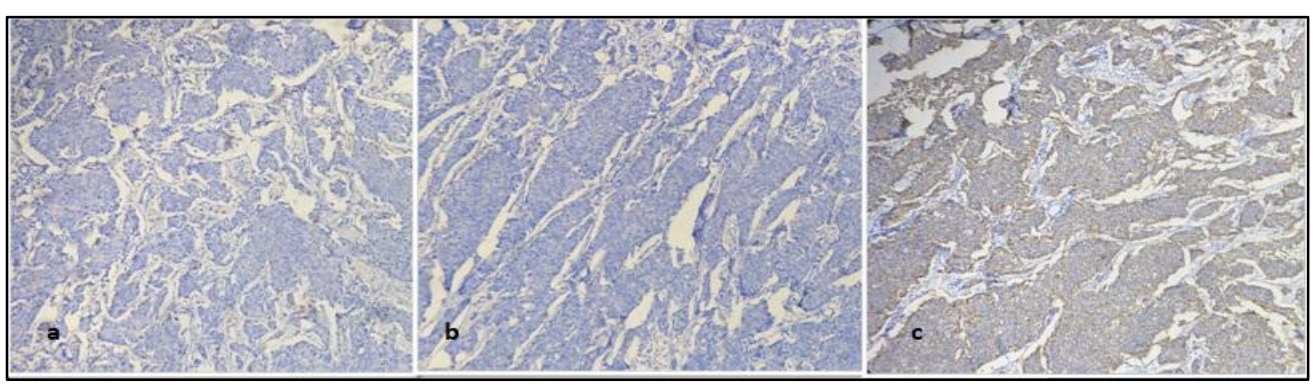

Fig-5: Immunohistochemistry in a breast carcinoma showing a) ER negative b) PR negative c) HER2/Neu positive 


\section{DISCUSSION}

Breast cancers have a heterogenous approach with varied implications for patients and physicians. Now days more and more targeted therapy towards molecular markers is being used in diagnosis and theuraptic measures. The advent of hormonal therapies has validated the distinction between ER positive and negative, like-wise for other hormones. The utilization of tamoxifen targeted therapy further added to our understanding. With introduction of trastuzumab (Herceptin) therapy the importance of amplified and overexpressed her 2/ Neu (HER 2) came into light [1]. Vici et al. first introduced the term "triple positive" breast cancer to describe a specific subtype [2]. They are defined as luminal HER2 tumor that expresses both ER and PR. In the current clinical settings, systemic theuraptic approaches for triple positive tumors comprise of hormone receptor (HR) specific hormonal therapies, targeted chemotherapy and HER 2 specific treatment [3]. The term triple negative is a group of cancers identified by absence of expression (ER), (PR) and absence of HER 2 over expression and amplification. This group comprise of $15-20 \%$ of all breast cancer types [4]. They have been characterized with clinicopathological features of younger age of onset, higher mean tumor size, and higher grade tumors and in few studies with higher rate of node positivity [4].

In the current study the total cases considered were 102 , the peak incidence was noted in the third and fourth decades with a median age of 48.9 yrs. The cases were broadly divided as triple negative, triple positive, double positive and single positive, the mean age of patients presenting in triple negative was $49.8 \mathrm{yrs}$. These findings are comparable to those by Liedtke et al. Dent et al. and Gaopande et al. [5-7]. On the basis of size of the tumor lesion most of the triple positive $(90.9 \%)$ were more than $5 \mathrm{cms}$ followed by triple negative cases (i.e. $81.8 \%$ ) and double positive $(78.8 \%)$. In other studies the mean tumor size more than $3 \mathrm{cms}$ is noted in triple negative cases than in triple positive which could be because the average size of the tumors that they considered were less than $3 \mathrm{cms}$ [6-8]. Significant association of necrosis was noted in triple negative cases $(63.6 \%)$ with $\mathrm{P}$ value of $(\mathrm{P}=0.0491)$ which was remarkably less in triple positive cases (27.3\%). Triple negative tumors have diverse morphology and basal cell features which help us to classify them in various groups, few of them like adenoid cystic and secretory carcinomas are well recognized and distinguishable. There are others which present with triple negative tumors with extensive comedo necrosis [9]. In contrast to apoptosis, comedo necrosis is the final result of bio energetic disability resulting due to ATP depletion to a level incompatible with cell survival, as an end result of toxic damage or physical insults. Glands with central lumen and devitalized cells usually present with comedo necrosis, especially in in-situ carcinoma (Figure 1). Although mitosis and necrosis have been recorded as histological feature of triple negative tumors its independent prognostic significance have been studied extensively $[9,11]$. Controversy exists regarding the definition and classification of necrosis with respect to amount of necrosis and relative distribution within intraductal and invasive components [11]. Various studies have interpreted that prognostic significance of tumor necrosis evaluation actually represents rapid growth rate which exceeds tumor sustaining angiogenesis to significant proportions [13, 14]. Majority of the triple negative are ductal in origin $[9,10]$ with several other phenotypes over represented including metaplastic, atypical or medullary. Infiltrating ductal carcinoma (NOS) was the most common diagnosis (90.6\%) followed by lobular mixed with ductal component, medullary and metaplastic carcinoma in the current study. Majority of the tumors were in grade II $(53 \%)$ and grade III $(25 \%)$ for the triple negative tumors. (Figure 2) In the single positive, double positive and triple positive most of the tumors were in Grade I $(48.5 \%)$. These findings were in accordance with the studies conducted by Dent et al. and others [6, 7, 12, 14]. Lympho-vascular invasion was noted more in triple positive cases $(63.6 \%$ and $36.4 \%)$ respectively. (Figure 3 ) In single positive cases vascular invasion (68\%) was noted more as compared to lymphatic invasion (56\%). (Figure 5) On the contrary triple positive lesions showed more lymphatic invasions $(63.6 \%)$ in contrast to vascular invasion (36.4\%). Triple negative had lymphatic invasions in $51.5 \%$ of cases and vascular invasion was noted in $60.6 \%$ of cases. These finding were in contrast to the studies conducted Gaopande et al. [7] but in accordance with studies done by Dent et al. and others $[6,15,16]$. Several studies have reported $40-50 \%$ metastasis in triple negative lesions as compared to other $[6,15,16]$. Ipsilateral metastatic deposits were $63.6 \%$ in triple positive and double positive lesions as compared to triple negative $(51.5 \%)$ and single positive lesions (48\%) in our study which is similar to findings of other studies (Figure 4).

\section{CONCLUSION}

Breast cancers are a varied group of disorders and generally thought, as being aggressive with poor prognosis. The management relies on traditional prognostic factors including nodal status, tumor histological grade and primary tumor size. It's absolutely important to corelate the clinical features with phenotypical expressions as course and disease prognosis will vary depending on the findings. In the current study we can conclude, that the parameters like size of the tumors, lympho-vascular invasion and lymphatic spread are variable factors and the prognosis and course of the tumor cannot be judged on the basis of these. Also the hormonal status as already assessed in other studies helps in defining the treatment modalities and disease progression of the same. However a close association has been noted between triple negative tumors and comedo necrosis, which 
definitely presents with different behavior and prognosis as compared to its absence. In fact, the presence of comedo necrosis in triple negative tumors is

\section{REFERENCES}

1. Perou, C. M., Sørlie, T., Eisen, M. B., Van De Rijn, M., Jeffrey, S. S., Rees, C. A., \& Fluge, Ø. (2000). Molecular portraits of human breast tumours. Nature, 406(6797), 747-752.

2. Vici, P., Pizzuti, L., Natoli, C., Gamucci, T., Di Lauro, L., Barba, M., \& Mariani, L. (2015). Triple positive breast cancer: a distinct subtype?. Cancer treatment reviews, 4l(2), 69-76.

3. Gradishar, W. J., Anderson, B. O., Balassanian, R., Blair, S. L., Burstein, H. J., Cyr, A., ... \& Goetz, M. P. (2017). NCCN guidelines insights: breast cancer, version 1.2017. Journal of the National Comprehensive Cancer Network, 15(4), 433-451.

4. Lehmann, B. D., Bauer, J. A., Chen, X., Sanders, M. E., Chakravarthy, A. B., Shyr, Y., \& Pietenpol, J. A. (2011). Identification of human triplenegative breast cancer subtypes and preclinical models for selection of targeted therapies. The Journal of clinical investigation, 121(7), 27502767.

5. Liedtke, C., Mazouni, C., Hess, K. R., André, F., Tordai, A., Mejia, J. A., \& Cristofanilli, M. (2008). Response to neoadjuvant therapy and long-term survival in patients with triple-negative breast cancer. Journal of clinical oncology, 26(8), 1275-1281.

6. Dent, R., Trudeau, M., Pritchard, K. I., Hanna, W. M., Kahn, H. K., Sawka, C. A., \& Narod, S. A. (2007). Triple-negative breast cancer: clinical features and patterns of recurrence. Clinical cancer research, 13(15), 4429-4434.

7. Gaopande, V. L., Joshi, S. S., Kulkarni, M. M., \& Dwivedi, S. S. (2015). A clinicopathologic study of triple negative breast cancer. Journal of the Scientific Society, 42(1), 12.

8. Albergaria, A., Ricardo, S., Milanezi, F., Carneiro, V., Amendoeira, I., Vieira, D., \& Schmitt, F. (2011). Nottingham Prognostic Index in triplenegative breast cancer: a reliable prognostic tool?. BMC cancer, 11(1), 299.

9. Kreike, B., van Kouwenhove, M., Horlings, H., Weigelt, B., Peterse, H., Bartelink, H., \& van de a good sign and they are documented to be clinically less aggressive. This helps in better assessment, management and overall better prognostic implications.

Vijver, M. J. (2007). Gene expression profiling and histopathological characterization of triplenegative/basal-like breast carcinomas. Breast Cancer Research, 9(5), R65.

10. Turner, N., Lambros, M. B., Horlings, H. M., Pearson, A., Sharpe, R., Natrajan, R., \& Ashworth, A. (2010). Integrative molecular profiling of triple negative breast cancers identifies amplicon drivers and potential therapeutic targets. Oncogene, 29(14), 2013-2023.

11. Kurebayashi, J., Moriya, T., Ishida, T., Hirakawa, H., Kirosumi, M., Akiyama, F. (2007). The prevalence of intrinsic subtypes and prognosis in breast cancer patients of different races. Breast.16 Suppl 2: 572-77.

12. Tsuda, H., Takarabe, T., Hasegawa, T., Murata, T., Hirohashi, S. (1999). Myoepithelial differentiation in high grade invasive ductal carcinoma with large central acellular zones. Hum Pathol; 30:1134-9.

13. Fulford, L.G., Easton, D.F., Reis, Filho, J.S., Sofronis, A., Gillett, C.E., Lakhani, S.R. (2006). Specific morphological features predictive for the basal phenotype in Grade\# invasive ductal carcinoma of breast. Histopathology; 49:22-34.

14. Jacquemier, J., Padovani, L., Rabayrol, L., Lakhani, S.R., Penault-llorca, F., Denoux, Y. (2005). Typical medullary carcinoma of the breast carcinoma has a basal/ myoepithelial phenotype. $J$ Pathol; 207:260-8.

15. Rakha, E.A., Elsheikh, S.E., Aleskandarany, M.A., HAbashi, H.O., \& green A.R., Powe, D.G. (2009). Triple negative breast cancer: Distinguishing between basal and nonbasal subtypes. Clin Cancer Res. 15:2302-10.

16. Rais, G., Raissouni, S., Aiteljah, M., Rais, F., Naciri, S. (2012). Triple negative breast cancers in Moroccan women: clinicipathological and theuraptic study at the national institute of oncology. BMC Womens Health. 12:35.

17. Lakhani, S.R., Ellis, I.O, Schnitt, S.J., Tan, P.H., Van, de Vijver., M.J., eds. (2012). WHO classification of Tumors of the breast. Fourth ed IARC, Lyon; ISBN 13. 